\title{
NÍVEIS DE FARELO DE COCO EM RAÇÕES PARA LEITÕES NA FASE DE CRECHE
}

\author{
LEVELS OF COPRA MEAL IN PIGLET DIETS AT NURSERY PHASE \\ Carvalho, L.E. ${ }^{*}$; Watanabe, P.H. ${ }^{\text {; }}$ Ribeiro, J.C. ${ }^{\text {; }}$ Nepomuceno, R.C. ${ }^{1}$; Gomes, T.R. ${ }^{1}$ \\ e Oliveira, E.L. ${ }^{1}$ \\ 'Departamento de Zootecnia. Centro de Ciências Agrárias. Universidade Federal do Ceará. Fortaleza-CE. \\ Brasil. *euquerio@ufc.br
}

\section{PalaVRAS ChaVE ADICIONAIS}

Alimento alternativo. Análise econômica. Desempenho zootécnico. Suíno.

\section{RESUMO}

Utilizou-se 32 leitões machos castrados e fêmeas de linhagem comercial, desmamados com 21 dias de idade e peso vivo médio de 6,20 $\pm 0,92$ $\mathrm{kg}$, objetivando avaliar o desempenho e a avaliação econômica de níveis inclusão de farelo de coco (FC) em rações para leitões na fase de creche. Os animais foram distribuídos em um delineamento em blocos ao acaso, com quatro tratamentos $(0,7,14$ e $21 \%$ de farelo de coco), oito repetições por tratamento e um animal por unidade experimental. Observou-se que dos 21 aos 42 dias de idade, a inclusão de níveis acima de $7 \%$ de farelo de coco na ração resultou em efeito linear negativo $(p<0,05)$ sobre o ganho de peso diário. Dos 43 aos 63 dias de idade e no período total (21 a 63 dias de idade), observou-se feito quadrático dos níveis de $\mathrm{FC}$ nas rações $(p<0,05)$ sobre a conversão alimentar, estimando-se melhor nível de 14,33 e 13,99\%, respectivamente. Para a fase dos 21 a 42 dias de idade, não houve efeito da inclusão de farelo de coco sobre as variáveis econômicas avaliadas. No entanto, dos 43 aos 63 dias e no período total (21 a 63 dias), observou-se efeito quadrático para as variáveis econômicas em função dos níveis de farelo de coco nas rações $(p<0,05)$, com melhores resultados ao nível estimado aproximado de $15 \%$. Recomenda-se a inclusão de até $7 \%$ de farelo de coco em rações para leitões dos 21 aos 42 dias de idade. Para a fase posterior, dos 43 a 63 dias de idade, o nível estimado de $15 \%$ resultou em melhores índices de desempenho e econômico. Recomenda-se a inclusão de até $7 \%$ de farelo de

\section{AdDitionAL KEYWORDS}

Alternative feedstuff. Economical analysis. Pigs. Zootechnical performance.

coco em rações para leitões dos 21 a 42 dias de idade. Para a fase posterior, dos 43 aos 63 dias de idade, o nível estimado de $15 \%$ resultou em melhores índices de desempenho.

\section{SUMMARY}

A total of 32 male castrated boars and gilts of a commercial pig line aged 21 days and $6.20 \pm 0.92$ $\mathrm{kg}$ of live weight were used to evaluate the performance and economical evaluation of copra meal inclusion in diets for piglets at nursery. Animals were allotted in a completely randomized block design with four treatments $(0,7,14$ and $21 \%$ of copra meal), eight replicates per treatment and one animal as experimental unit. From 21 to 42 days of age, levels up to $7 \%$ reduced daily weight gain of piglets $(p<0.05)$. A quadratic trend on feed:gain ratio was observed from 42 to 63 days and total period $(p<0.05)$, with better results at estimated level of 14.33 and $13.99 \%$, respectively. No effect of copra meal inclusion on economical variables was observed from 21 to 42 days of age. From 43 to 63 days and total period ( 21 to 63 days) a quadratic effect on economical indexes was observed with the inclusion of different levels of copra meal, with better results around $15 \%$. The inclusion of copra meal until the level of $7 \%$ is recommended in piglet diets from 21 to 42 days of age. From 43 to 63 days, the estimated level of $15 \%$ results in better performance and economical indexes. It is recommended to include up to $7 \%$ of 


\section{CARVALHO, WATANABE, RIBEIRO, NEPOMUCENO, GOMES E OLIVEIRA}

coconut meal in diets for pigs from 21 to 42 days old. From 43 to 63 days of age, the estimated level of $15 \%$ resulted in better performance.

\section{INTRODUÇÃO}

Nos sistemas de produção intensiva de suínos, o desmame precoce dos leitões, entre 21 e 28 dias de idade, tornou-se uma prática comum, visto que permite a obtenção de maior produtividade das matrizes. Todavia, a fase de creche, para leitões desmamados precocemente, exige atenção especial direcionada aos manejos sanitário, alimentar e nutricional, uma vez que os sistemas imunológico e digestivo dos animais encontram-se imaturos.

Diante do desafio nutricional no período pós-desmame, a alternativa viável para obtenção de bom desempenho dos animais tem sido o fornecimento de rações com altas digestibilidade e palatabilidade e com densidades nutricional e energética adequadas às exigências dos leitões. As rações complexas, compostas por ingredientes de alta digestibilidade, têm sido consideradas adequadas para leitões em fase de creche.

Todavia o custo desse tipo de ração é elevado, principalmente em virtude da dependência do milho e da soja, cuja produção é desfavorável em regiões não produtoras de grãos, o que pode reduzir a competitividade da produção de suínos. Nesse contexto, observa-se a possibilidade de uso subprodutos da agroindústria na alimentação de suínos, sendo estes geralmente utilizados nas fases de crescimento e terminação, embora também seja possível a inclusão em dietas para animais na fase de creche.

Na Região Nordeste do Brasil, dentre os ingredientes alternativos, destaca-se o farelo de coco, subproduto obtido da extração do óleo de coco. De acordo com AGRIANUAL (2006) a região costeira nordestina é responsável por $96 \%$ da produção nacional de cocos, e grande parte é destinada à indústria de alimentos. Nesse sentido, o farelo de coco pode ser utilizado como fonte alternativa de energia e proteína na alimentação de suínos, substituindo parcialmente o milho e o farelo de soja nas rações. De acordo com a tabela de composição de alimentos da EMBRAPA (1991), o farelo de coco apresenta $27,37 \%$ de proteína bruta $(\mathrm{PB}), 14,20 \%$ de extrato etéreo (EE), $12,26 \%$ de fibra bruta (FB) e $3096 \mathrm{kcal}$ de energia metabolizável (EM/kg) para suínos. Segundo Rostagno et al. (2011), esse subproduto contém $21,85 \%$ de PB, $2885 \mathrm{kcal}$ de EM/kg para suínos; 3,15\% de EE, e 52,35 $\%$ de fibra em detergente neutro (FDN) e $27,10 \%$ de fibra em detergente ácido (FDA). As variações na composição e no valor nutricional do farelo de coco decorrem do tipo de processamento utilizado, que influencia principalmente a quantidade de gordura (Panigrahi, 1989).

A proteína do farelo de coco pode ser considerada de qualidade inferior à do farelo soja, em função do baixo coeficiente de digestibilidade para suínos $(67,3 \%)$ e em virtude da deficiência em lisina e baixos valores dos demais aminoácidos essenciais (Rostagno et al., 2011). Além disso, os elevados teores de fibras, bem como, as altas concentrações de mananos na fração fibrosa deste subproduto podem interferir na digestão e absorção dos nutrientes e reduzir seu aproveitamento, podendo ser um limitante da sua inclusão nas rações para animais monogástricos (Pascoal et al., 2010).

Thorne et al. (1992), O'Doherty e McKeon, (2000) e Siebra et al. (2009) observaram que a inclusão de até $20 \%$ de farelo de coco em dietas para suínos em crescimento e terminação não afetou desempenho e a digestibilidade das dietas, expressando o potencial nutritivo do farelo de coco. Assim, objetivou-se avaliar o efeito de crescentes níveis de farelo de coco em dietas para leitões desmamados sobre o desempenho e avaliação econômica.

\section{MATERIALEMÉTODOS}

O experimento foi realizado no Setor de Suinocultura do Departamento de Zootec- 
nia do Centro de Ciências Agrárias da Universidade Federal do Ceará (DZ/CCA/ UFC), situado no Campus do Pici, em Fortaleza-CE. Foram utilizados 32 leitões fêmeas e machos castrados, de linhagem comercial, desmamados com média de 21 dias de idade e peso vivo médio de $6,20 \pm 0,92 \mathrm{~kg}$.

$\mathrm{O}$ experimento foi conduzido durante o período de creche dos leitões, dividido em duas fases: 21 a 42 dias de idade e 43 a 63 dias de idade.

Os leitões foram alojados em um galpão de alvenaria, cobertura com telhas de barro e piso compacto de cimento (rugosidade média) tendo $3 \%$ de declividade, composto por 32 baias, distribuídas ao longo de um corredor central, medindo, cada uma, 3,00 x $1,50 \mathrm{~m}$ e equipadas com um comedouro de cimento e um bebedouro tipo chupeta, instalados em extremidades opostas.

Os animais foram distribuídos seguindo um delineamento em blocos casualizados, com quatro tratamentos $(0,7,14$ e $21 \%$ de farelo de coco) e oito repetições por tratamento, sendo a unidade experimental constituída por um animal. O peso individual dos animais ao inicio da pesquisa foi o critério utilizado para a formação dos blocos.

A temperatura do galpão foi monitorada diariamente, às 08 e 17 h, por meio de dois termohigrômetros, instalados no interior do galpão, na altura dos animais.

$\mathrm{O}$ farelo de coco utilizado nesse estudo foi obtido de uma indústria de beneficiamento de coco (DuCôco), localizada no município de Itapipoca no Estado do Ceará. As amostras de farelo de coco bem como dos demais ingredientes foram realizadas no Laboratório de Nutrição Animal do Departamento de Zootecnia da UFC, onde foram determinados os valores de matéria seca e proteína bruta. Os valores da energia metabolizável, extrato etéreo, cálcio, fósforo, sódio, lisina, metionina e amido foram obtidos das tabelas Rostagno et al. (2005) e corrigidos de acordo com o teor de matéria seca encontrados.

As rações experimentais utilizadas (tabela I) foram formuladas para serem isonutrientes, considerando-se as exigências nutricionais para leitões de 7 a $15 \mathrm{~kg}$ e de 15 a $30 \mathrm{~kg}$, de acordo com as recomendações de Rostagno et al. (2005). As rações e a água foram fornecidas aos animais à vontade.

Os dados referentes a ganho de peso diário e consumo de ração diário foram obtidos por pesagens semanais individuais dos animais e de suas respectivas rações, menos os resíduos presentes nos comedouros. A partir destes dados, calculou-se a conversão alimentar.

Os valores dos ingredientes utilizados na elaboração dos custos foram obtidos na região metropolitana de Fortaleza, sendo ${ }^{1}$ : milho ( $\mathrm{R} \$ 0,63 / \mathrm{kg})$, farelo de soja ( $\mathrm{R} \$ 0,98 /$ $\mathrm{kg})$, farelo de coco $(\mathrm{R} \$ 0,40 / \mathrm{kg})$, leite em pó desnatado ( $\mathrm{R} \$ 17,97 / \mathrm{kg})$, açúcar ( $\mathrm{R}$ \$ 0,99/ $\mathrm{kg}$ ), óleo de soja (R \$2,82/L), fosfato bicálcico ( $\mathrm{R} \$ 3,00 / \mathrm{kg})$, calcário ( $\mathrm{R} \$ 0,15 / \mathrm{kg})$, sal comum ( $\mathrm{R} \$ 0,18 / \mathrm{kg}), \mathrm{L}-$ lisina $\mathrm{HCl}(\mathrm{R} \$ 8,00 / \mathrm{kg}), \mathrm{DL}-$ metionina ( $\mathrm{R} \$ 20,00 / \mathrm{kg}$ ), suplemento vitamínico-mineral ( $\mathrm{R} \$ 0,43 / \mathrm{kg})$.

Para verificar a avaliação econômica dos diferentes níveis de inclusão do farelo de coco nas rações, determinou-se inicialmente o custo da ração (CR) por quilograma de peso vivo ganho (Yi), segundo a equação proposta por Bellaver et al. (1985):

$$
Y_{i}=\left(Q_{i} \times P_{i}\right) / G_{i}
$$

em que:

$Y_{i}=$ custo da ração por quilograma de peso vivo ganho no i-ésimo tratamento;

$\mathrm{P}_{\mathrm{i}}=$ preço por quilograma da ração utilizada no $\mathrm{i}-$ ésimo tratamento;

$Q_{i}=$ quantidade de ração consumida no i-ésimo tratamento:

$G_{i}=$ ganho de peso do i-ésimo tratamento.

Em seguida, foram calculados o índice de eficiência econômica (IEE) e o índice de

${ }^{1}$ Dólar comercial em março de 2013 cotado a $\mathrm{R} \$ 1,79$. 


\section{CARVALHO, WATANABE, RIBEIRO, NEPOMUCENO, GOMESE OLIVEIRA}

Tabela I . Composição, nutricional e custos das rações experimentais. (Nutritional composition and cost of the experimental diets).

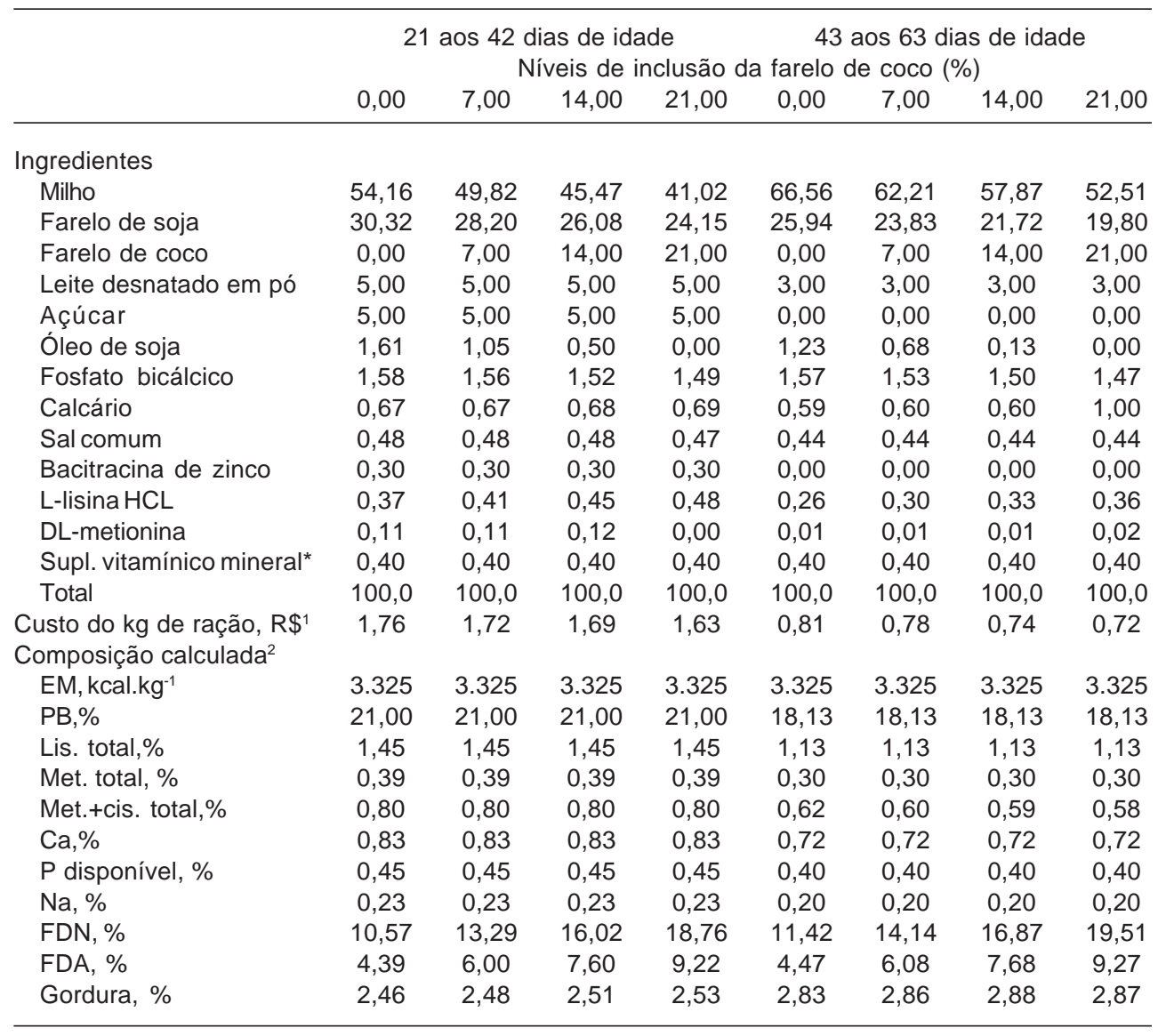

*Suplemento vitamínico e mineral. Quantidade por kg do produto: $1500000 \mathrm{UI}$ de Vit. A, $450000 \mathrm{UI}$ de Vit. D3, $7500 \mathrm{mg}$ de Vit. E, $1500 \mathrm{mg}$ de Vit. K, $3250 \mathrm{mg}$ de Vit. B1, $1300 \mathrm{mg}$ de Vit. B2, $375 \mathrm{mg}$ de Vit. B6, $5000 \mathrm{mcg}$ de Vit. B12, $7500 \mathrm{mg}$ de niacina, $4500 \mathrm{mg}$ de pantotenato de cálcio, $150 \mathrm{mg}$ de ácido fólico, $22,50 \mathrm{mg}$ de biotina, $68000 \mathrm{mg}$ de colina, $12500 \mathrm{mg}$ de ferro, $5250 \mathrm{mg}$ de cobre, $8750 \mathrm{mg}$ de manganês, $26250 \mathrm{mg}$ de zinco, $350 \mathrm{mg}$ de lodo, $75 \mathrm{mg}$ de selênio, $1000 \mathrm{mg}$ de antioxidante.

'Dólar comercial em março de 2013 cotado a $R \$ 1,79$.

${ }^{2}$ Composição calculada com base nas informações contidas em Rostagno et al. (2005).

custo (IC) propostos por Fialho et al. (1992):

$$
\begin{aligned}
& \mathrm{IEE}=(\text { MCei } / \text { CTei }) \times 100 \mathrm{e} \\
& \mathrm{IC}=(\text { CTei } / \mathrm{MCei}) \times 100
\end{aligned}
$$

onde:

MCei= Menor custo da ração por quilograma ganho, observado entre tratamentos; CTei= Custo do tratamento i considerado.

A análise estatística foi realizada utilizando-se o software Statistical Analyses System (SAS, 2001). Os dados de desem-

Archivos de zootecnia vol. 63, núm. 242, p. 298. 


\section{NÍVEIS DE FARELO DE COCO EM RAÇÕES PARA LEITÕES NA FASE DE CRECHE}

penho zootécnico e avaliação econômica tiveram os graus de liberdade, referentes aos níveis de inclusão do farelo de coco, excluindo-se a ração testemunha, desdobrados em polinômios, para estabelecer a curva que melhor descrevesse o comportamento dos dados. Para comparação dos resultados obtidos com cada um dos níveis de inclusão do farelo de coco em relação à ração controle, foi utilizado o teste de Dunnett, a $5 \%$ de significância.

\section{RESULTADOSEDISCUSSÃO}

A temperatura ambiental média no interior do galpão durante o período experimental foi de $26,9{ }^{\circ} \mathrm{C}$, sendo as médias registradas de temperatura máxima e mínima de 30,7 e $23,1^{\circ} \mathrm{C}$, respectivamente e $74 \%$ de umidade relativa do ar. Também foi registrada nesse período uma precipitação pluviométrica de $50,9 \mathrm{~mm}$.

Pelo teste de comparação de médias, observou-se que não houve diferença $(\mathrm{p}>0,05)$ entre os resultados obtidos para as rações contendo $\mathrm{FC}$ em relação à ração controle em nenhuma das fases estudadas de desempenho zootécnico.

$\mathrm{Na}$ análise de regressão dos dados (tabela II), foi observado que não houve efeito significativo para a inclusão de níveis crescentes do FC sobre o consumo de ração diário e a conversão alimentar dos 21 aos 42 dias de idade, porém houve efeito linear $(\mathrm{p}<0,05)$ sobre o ganho de peso diário, estimando-se que o nível de $7 \%$ de inclusão do FC resultou em maior ganho, onde de acordo com a equação obtida, a partir desse nível de inclusão, a cada $1 \%$ adicional de FC resultaria na redução de 4,83 (g/leitão) nesta fase.

A redução no ganho de peso com o acréscimo de FC acima de $7 \%$ observada na análise de regressão pode ter ocorrido devido à ação de uma barreira física imposta pela lignina (Fukushima e Dehority, 2000),

Tabela II. Valores médios e equações de regressão para consumo de ração diário (CRD), ganho de peso diário (GPD) e conversão alimentar (CA) dos leitões na fase de creche alimentados com rações contendo níveis crescentes de inclusão de farelo de coco. (Mean values and regression equations for feed intake (CRD), daily weight gain (GPD) and feed conversion (CA) of piglets at nursery phase fed diets containing increasing levels of coconut meal).

\begin{tabular}{|c|c|c|c|c|c|c|c|}
\hline \multirow[t]{2}{*}{ Variáveis } & \multicolumn{4}{|c|}{ Níveis de farelo de coco (\%) } & \multirow[t]{2}{*}{ CV (\%) } & \multirow[t]{2}{*}{ Regressão } & \multirow[t]{2}{*}{$p$} \\
\hline & 0 & 7 & 14 & 21 & & & \\
\hline \multicolumn{8}{|c|}{21 a 42 dias de idade } \\
\hline CRD (kg) & $0,4180^{\mathrm{a}}$ & $0,4467^{a}$ & $0,3472^{\mathrm{a}}$ & $0,3502^{a}$ & 12,72 & NS & 0,1165 \\
\hline GPD (kg) & $0,2733^{a}$ & $0,2987^{a}$ & $0,2251^{\mathrm{a}}$ & $0,2310^{\mathrm{a}}$ & 13,66 & Linear $^{1}$ & 0,1397 \\
\hline CA & $1,60^{\mathrm{a}}$ & $1,49^{a}$ & $1,56 a$ & $1,50^{\mathrm{a}}$ & 3,37 & NS & 0,7270 \\
\hline \multicolumn{8}{|c|}{43 a 63 dias de idade } \\
\hline CRD (kg) & $0,9236^{a}$ & $0,9677^{a}$ & $0,8641^{a}$ & $0,8830^{\mathrm{a}}$ & 5,05 & NS & 0,5173 \\
\hline GPD (kg) & $0,4712^{a}$ & $0,4802^{a}$ & $0,4946^{a}$ & $0,4445^{a}$ & 4,46 & NS & 0,6742 \\
\hline CA & $1,95^{\mathrm{a}}$ & $2,02^{\mathrm{a}}$ & $1,76 a$ & $1,97^{\mathrm{a}}$ & 5,91 & Quadrático² & 0,0143 \\
\hline \multicolumn{8}{|l|}{ Período total } \\
\hline CRD (kg) & $0,6690^{a}$ & $0,7075^{a}$ & $0,6056^{a}$ & $0,6167^{a}$ & 7,29 & NS & 0,2484 \\
\hline GPD (kg) & $0,3722^{a}$ & $0,3925^{a}$ & $0,3598^{a}$ & $0,3380^{a}$ & 6,24 & NS & 0,4584 \\
\hline CA & $1,80^{\mathrm{a}}$ & $1,81^{\mathrm{a}}$ & $1,69^{\mathrm{a}}$ & $1,81^{\mathrm{a}}$ & 3,29 & Quadrático ${ }^{3}$ & 0,1647 \\
\hline
\end{tabular}

$C V=$ coeficiente de variação; NS= não significativo $(p>0,05)$ pelo teste de Dunnett.

${ }^{1} Y=-0,00483 x+0,3193 ; R^{2}=0,26 ;{ }^{2} Y=0,00478 x^{2}-0,13709 x+2,7460 ; R^{2}=0,33 ;{ }^{3} Y=0,00240 x^{2}-0,06718 x$ $+2,1672 ; R^{2}=0,17$ 


\section{CARVALHO, WATANABE, RIBEIRO, NEPOMUCENO, GOMES E OLIVEIRA}

promovido pelo incremento do teor de fibra na dieta que resultou no aumento de $8,11 \mathrm{e}$ $8,09 \%$ no teor de FDN da ração controle para o tratamento com maior nível de inclusão nas fases dos 21 aos 42 dias de idade e dos 43 aos 63 dias de idade, respectivamente.

Entre os efeitos do acréscimo da fibra na alimentação de suínos são comuns os relatos de redução no aproveitamento de nutrientes da ração, diminuindo a sua energia metabolizável, com consequente redução na taxa de crescimento (Ferreira, 1994) e pelo fato dos animais jovens provavelmente ainda não apresentarem trato digestivo adaptado a dietas com esta característica (Costa et al., 2005). Entre outros efeitos associativos ao nível de incorporação da fibra insolúvel sobre leitões cita-se a influência nos processos digestivos como a irritação da mucosa intestinal por abrasão mecânica, levando a aumentos nas secreções endógenas de muco e água, que podem influenciar a digestão e absorção de outros nutrientes, sendo o efeito negativo causado pela proteção de alguns nutrientes à ação enzimática no intestino delgado (Wenk, 2001; Montagne et al., 2003; Hetland et al., 2004).

Bastos et al., 2007, ao avaliarem a inclusão de FC em dietas para frangos de corte também observaram que a utilização deste ingrediente não deve ser superior a $5 \%$ na fase de 7 aos 21 dias de idade, devido a maior susceptibilidade das aves jovens aos efeitos negativos do elevado nível de fibra na ração, havendo menor efeito com o aumento da idade das aves. Esse comportamento adaptativo do sistema digestivo se assemelha ao dos leitões na fase inicial, em que o aproveitamento das frações fibrosas melhora proporcionalmente com a maturidade fisiológica do animal.

De acordo com a análise de regressão dos dados referentes ao período de 43 aos 63 dias de idade e do período total (21 aos 63 dias de idade) (tabela II), foi observado que houve efeito quadrático dos níveis de FC nas rações $(p<0,05)$ sobre a conversão alimentar. Analisando as equações obtidas, estimou-se que a conversão alimentar melhorou com a inclusão de $14,33 \%$ do FC na fase inicial II e 13,99\% no período total.

Percebe-se que a inclusão de níveis acima de $14 \%$ de farelo de coco nas dietas para leitões com idade a partir de 42 dias acarreta em piora na conversão alimentar. De acordo com Castillo-Soto et al. (2004), observa-se que os níveis de enzimas endógenas se reduzem por ocasião do desmame, havendo uma recuperação variável em função principalmente da idade. Assim, aos 42 dias de idade, os leitões já apresentam maior atividade enzimática no trato digestório, o que possibilitaria maior nível de inclusão do FC em relação à fase inicial.

A demanda nutricional de leitões até o final da fase de creche é correspondente a ingredientes com alta densidade energética e reduzido teor de fibroso (Bellaver e Ludke, 2004). Assim é pouco usual que se faça a inclusão de ingredientes que possam elevar o teor de fibra da ração, como é o caso do FC. No entanto, por meio dos resultados obtidos atenta-se que há possibilidade de inclusão deste ingrediente em substituição parcial ao milho e farelo de soja, apresentando-se mais viável a partir dos 43 dias de idade, momento o qual os leitões já se encontram com maior maturidade fisiológica.

Em relação à avaliação econômica (tabela III), para a fase dos 21 aos 42 dias de idade, não houve efeito da inclusão de farelo de coco sobre as variáveis econômicas avaliadas.

Já dos 43 aos 63 dias e no período total (21 aos 63 dias), observou-se efeito quadrático para as variáveis econômicas em função dos níveis de $\mathrm{FC}$ nas rações $(\mathrm{p}<0,05)$. De acordo com as equações obtidas, estimou-se que dos 43 aos 63 dias de idade, o custo da ração por quilograma de ganho de peso vivo (CRPV) e os índices de custo (IC) reduziram até a inclusão de aproximadamente $15,49 \%$ de FC enquanto que o índice de eficiência econômica (IEE) melhorou com o nível estimado de 15,24\%. Para o período total, os níveis ótimos estimados de 


\section{NÍVEIS DE FARELO DE COCO EM RAÇÕES PARA LEITÕES NA FASE DE CRECHE}

inclusão do FC foram 15,$77 ; 15,72$ e 15,67\%, para CRPV, IC e IEE, respectivamente.

Pelo teste das médias foi observado que a inclusão de $14 \%$ do FC resultou em menor custo com ração a cada quilo de ganho de peso vivo do suíno, menor índice de custo da ração e melhor índice de eficiência econômica em relação à ração controle para o período de 43 aos 63 dias de idade e período total.

Os níveis de 7 e $14 \%$ de inclusão do FC nas rações para a fase inicial são inferiores aos recomendados por Siebra et al. (2008), que ao avaliarem níveis de $0,10,20$ e $30 \%$ de inclusão deste ingrediente nas dietas de suínos em crescimento e terminação, verificaram maior retorno econômico com a inclusão de 22,5\% de FC em dietas à base de milho e farelo de soja. Esses resultados também discordaram dos encontrados por O'Doherty e McKeon (2000), que trabalhando com suínos nas fases de crescimento e terminação, utilizando dois níveis (20 e $40 \%$ ) de FC, afirmaram que quanto maior o nível de inclusão deste ingrediente, menores são os custos. Entretanto para leitões na fase inicial, que são animais mais sensíveis à presença de ingredientes fibrosos como o farelo de coco, esses níveis encontrados na presente pesquisa, permitem a elaboração de rações mais viáveis economicamente, respeitando os limites fisiológicos da categoria.

A partir dos 43 dias de idade, o farelo de coco pode ser incluídos até o nível de $15 \%$ sem afetar o desempenho dos animais, sendo economicamente favorável sua inclusão em dietas para leitões.

Embora não tenha havido diferença significativa na fase inicial I, a inclusão de FC até o nível de $21 \%$ não é recomendada para essa primeira fase, pois como visto na análise de desempenho, a utilização de níveis de FC acima de $7 \%$ influenciou negativamente o

Tabela III. Custo com ração por quilograma de peso vivo ganho (CR), índice de custo da ração (IC) e índice de eficiência econômica (IEE) de leitões na fase de creche alimentados com diferentes níveis de farelo de coco. (Cost of feed per kilogram live weight gain (CR), feed cost index (IC) and economic efficiency index (IEE) of piglets in nursery phase fed different levels of coconut meal).

\begin{tabular}{|c|c|c|c|c|c|c|c|}
\hline \multirow[t]{2}{*}{ Variáveis } & \multicolumn{4}{|c|}{ Níveis de farelo de coco (\%) } & \multirow[t]{2}{*}{ CV (\%) } & \multirow[t]{2}{*}{ Regressão } & \multirow[t]{2}{*}{$p$} \\
\hline & 0 & 7 & 14 & 21 & & & \\
\hline \multicolumn{8}{|c|}{21 a 42 dias de idade } \\
\hline $\mathrm{CR}(\mathrm{R} \$ / \mathrm{kg})$ & $2,8229^{a}$ & $2,5719^{a}$ & $2,6520^{a}$ & $2,4544^{a}$ & 5,89 & NS & 0,1165 \\
\hline IC (\%) & $191,94^{a}$ & $182,58^{\mathrm{a}}$ & $188,26^{a}$ & $184,71^{\mathrm{a}}$ & 2,20 & NS & 0,7288 \\
\hline IEE (\%) & $51,059^{a}$ & $55,366^{a}$ & $53,391^{a}$ & $54,369^{a}$ & 3,44 & NS & 0,5225 \\
\hline \multicolumn{8}{|c|}{43 a 63 dias de idade } \\
\hline $\mathrm{CR}(\mathrm{R} \$ / \mathrm{kg})$ & $1,5832^{a}$ & $1,5983^{a}$ & $1,3053^{c}$ & $1,4230^{b}$ & 9,44 & Quadrático ${ }^{1}$ & 0,5173 \\
\hline IC (\%) & $133,55^{a}$ & $134,83^{a}$ & $110,11^{c}$ & $120,04^{b}$ & 9,44 & Quadrático² & 0,0001 \\
\hline IEE (\%) & $75,063^{a}$ & $74,885^{a}$ & $91,590^{c}$ & $83,635^{b}$ & 9,82 & Quadrático ${ }^{3}$ & 0,0001 \\
\hline \multicolumn{8}{|l|}{ Período total } \\
\hline $\mathrm{CR}(\mathrm{R} \$ / \mathrm{kg})$ & $2,3245^{a}$ & $2,2692^{\mathrm{a}}$ & $2,0617^{c}$ & $2,1324^{b}$ & 5,50 & Quadrático 4 & 0,2484 \\
\hline IC (\%) & $127,28^{a}$ & $124,25^{a}$ & $112,88^{c}$ & $116,76^{b}$ & 5,51 & Quadrático 5 & 0,0049 \\
\hline $\operatorname{IEE}(\%)$ & $78,744^{a}$ & $80,788^{a}$ & $89,004^{c}$ & $86,068^{b}$ & 5,63 & Quadrático 6 & 0,0052 \\
\hline
\end{tabular}

$C V=$ coeficiente de variação; NS= não significativo $(p>0,05)$ pelo teste de Dunnett a $5 \%$ de significância. ${ }^{1} Y=0,00419 x^{2}-0,12985 x+2,30203 ; R^{2}=0,48 ;{ }^{2} Y=0,35349 x^{2}-10,95438 x+194,1909 ; R^{2}=0,48 ;{ }^{3} Y=$ $0,25162 x^{2}-7,67068 x+33,5200 ; R^{2}=0,47 ;{ }^{4} Y=0,00283 x^{2}-0,08927 x+2,7549 ; R^{2}=0,26 ;{ }^{5} Y=0,15546 x^{2}$ $-4,88802 x+150,8504 ; R^{2}=0,26 ;{ }^{6} Y=0,1137 x^{2}-3,56342 x+61,4209 ; R^{2}=0,25$. 


\section{CARVALHO, WATANABE, RIBEIRO, NEPOMUCENO, GOMESE OLIVEIRA}

ganho de peso diário dos leitões.

\section{CONCLUSÕES}

Recomenda-se a inclusão de até $7 \%$ de

\section{BIBLIOGRAFIA}

AGRIANUAL. 2006. Anuário da agricultura brasileira. Coco-da-baía. FNP, Consultoria e Agroinformativos. São Paulo. pp. 286-292.

Bastos, S.C.; Fuentes, M.F.F.; Freitas, E.R.; Espindola, G.B. e Braga, C.V.P. 2007. Efeito da inclusão do farelo de coco em rações para frangos de corte. Rev Ciênc Agron, 38: 297303.

Bellaver, C.; Fialho, E.T.; Protas, J.F.S. e Gomes, P.C. 1985. Radícula de malte na alimentação de suínos em crescimento e terminação. Pesqui Agropecu Bras, 20: 969-74.

Bellaver, C. e Ludke, J.V. 2004. Considerações sobre os alimentos alternativos para dietas de suínos. In: Encontro Internacional dos Negócios da Pecuária. Anais... ENIPEC. Cuiabá, MS.

Castillo-Soto, W.L.; Kronka, R.N.; Pizauro Jr., J.M.; Thomaz, M.C. e Carvalho, L.E. 2004. Efeito da substituição do farelo de soja pela levedura (Saccharomyces cerevisiae) como fonte protéica em dietas para leitões desmamados sobre a morfologia intestinal e atividade das enzimas digestivas intestinais. Arch Latinoam Prod Anim, 12: 21-27.

Costa, M.C.R.; Silva, C.A.; Pinheiro, J.W.; Fonseca, N.A.N.; Souza, N.E.; Visentainer, J.V.; Belé, J.C.; Borosky, J.C.; Murinho, F.L. e Agostini, P.S. 2005. Utilização da torta de girassol na alimentação de suínos nas fases de crescimento e terminação: Efeitos no desempenho e nas características de carcaça. Rev Bras Zootecn, 34: 1581-1588.

EMBRAPA. 1991. Empresa Brasileira de Pesquisa e Agropecuária. Concórdia. Circular Técnica, 19. pp. 28-68.

Ferreira, W.M. 1994. Os componentes da parede celular vegetal na nutrição de não-ruminantes. Reunião Anual da Sociedade Brasileira de Zootecnia, 31. Simpósio Internacional de Produção de Não-ruminante. Anais... EDUEM. Maringá. pp. 85-113.

Fialho, E.T.; Barbosa, O.; Ferreira, A.S. e Gomes, farelo de coco em rações para leitões dos 21 a 42 dias de idade. Para a fase posterior, dos 43 aos 63 dias de idade, o nível estimado de $15 \%$ resultou em melhores índices de desempenho zootécnico e econômico.

P.C. 1992. Utilização da cevada suplementada com óleo de soja para suínos em crescimento e terminação. Pesqui Agropecu Bras, 27: 14671475.

Fukushima, R.S. and Dehority, B.A. 2000. Feasibility of using lignin isolated from forages by solubilization in acetyl bromide as a standard for lignin analysis. J Anim Sci, 78: 3135-3143.

Hetland, H.; Choct, M. and Svihus, B. 2004. Role of insoluble non-starch polysaccharides in poultry nutrition. World Poultry Sci J, 60: 415-422.

Montagne, L.; Pluske, J.R. and Hampson, D.J. 2003. A review of interactions between dietary fibre and the intestinal mucosa, and their consequences on digestive health in young non-ruminant animals. Anim Feed Sci Tech, 108: 95-117.

O'Doherty, J.V. and McKeon, M.P. 2000. The use of expeller copra meal in grower and finisher pig diets. Livest Prod Sci, 67: 55-65.

Panigrahi, S. 1989. Effects on egg production of including high residual lipid copra meal in laying hen diets. Brit Poultry Sci, 30: 305-312.

Pascoal, L.A.F.; Miranda, E.C.; Lamenha, M.I.A.; Watanabe, P.H.; Miranda, C.C.; Silva, L.P.G. e Araújo, D.M. 2010. Inclusão de farelo de coco em dietas para suínos em crescimento com ou sem suplementação enzimática. Rev Bras Saúde Prod Anim, 11: 160-169.

Rostagno, H.S.; Albino, L.F.T.; Donzele, J.L.; Gomes, P.C.; Oliveira, R.F.; Lopes, D.C.; Ferreira, A.S. e Barreto, S.L.T. 2005. Tabelas brasileiras para aves e suínos: composição de alimentos e exigências nutricionais. 2. ${ }^{\text {a }}$ ed. UFV. ViçosaMG. 186 pp.

Rostagno, H.S.; Albino, L.F.T.; Donzele, J.L.; Gomes, P.C.; Oliveira, R.F.; Lopes, D.C.; Ferreira, A.S. e Barreto, S.L.T. 2011. Tabelas brasileiras para aves e suínos: composição de alimentos

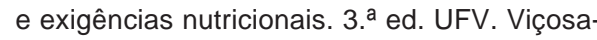
MG. 252 pp.

Siebra, J.E.C.; Ludke, M.C.M.M.; Ludke, J.V.;Bertol,

Archivos de zootecnia vol. 63, núm. 242, p. 302. 


\section{NÍVEIS DE FARELO DE COCO EM RAÇÕES PARA LEITÕES NA FASE DE CRECHE}

T.M. e Dutra Júnior, W.M. 2008. Uso de farelo de coco nas dietas de suínos para abate. Rev Bras Saúde Prod Anim, 10: 604-614.

SAS. 2001. Statistical Analysis System. System for Microsoft Windows. Release 8.2. SAS Inst. Cary.

Thorne, P.J.; Wiseman, J.; Cole, D.J.A. and Machin, D.H. 1992. Effects of level of inclusion of copra meal in balanced diets supplemented with synthetic amino acids on growth and fat deposition and composition in growing pigs fed ad libitum at a constant temperature of $25 \stackrel{\circ}{\circ}$. Anim Feed Sci Tech, 40: 31-40.

Wenk, C. 2001. The role of dietary ûbre in the digestive physiology of the pig. Anim Feed $\mathrm{SCl}$ Tech, 90: 21-33. 